\title{
GAIA Level 2b Failure to Thrive
}

National Cancer Institute

\section{Source}

National Cancer Institute. GAIA Level 2 b Failure to Thrive. NCI Thesaurus. Code

C128772.

GAIA Level 2b Failure to Thrive is defined by either of two criteria: first, the following four requirements must be present: a) Infant age determined by a documented birth date; b) Weights are obtained using a spring balance scale; c) A minimum of two weights measured at least four weeks apart; d) Weight for age deceleration must pass through at least two centile spaces on a growth chart; OR second, the following three requirements must be present: a) Weight is measured using an electronic scale or beam balance scale; b) Length is documented using infantometer; c) Weight for length ratio less than or equal to the third centile on an appropriate growth chart. 